\title{
Florais de Bach na Odontologia: uma breve revisão da literatura
}

\author{
Bach Florals in Dentistry: a brief literature review
}

Flores de Bach en Odontología: una breve revisión de la literatura

\section{Ayra Raissa da Silva SANTOS ${ }^{1}$} Laís Sousa MAIA ${ }^{1}$

Luiza Queiroz Rosado de SOUZA ${ }^{1}$

Heloísa Mara Batista Fernandes de OLIVEIRA²

Elizandra Silva da PENHA ${ }^{3}$

Abrahão Alves de OLIVEIRA FILHO ${ }^{4}$

${ }^{1}$ Curso de Odontologia, Universidade Federal de Campina Grande UFCG 58708-110, Patos, Paraíba, Brasil

2 Professora da Universidade Federal do Rio Grande do Norte UFRN 59200-000, Santa Cruz, Rio Gande do Norte, Brasil ${ }_{3}$ Professora da Universidade Federal de Campina Grande UFCG 58708-110, Patos, Paraíba, Brasil

${ }^{4}$ Professor Doutor, Unidade Acadêmica de Ciências Biológicas, Centro de Saúde e Tecnologia Rural, Universidade Federal de Campina Grande,UFCG 58428-830 Campina Grande - PB, Brasil

\section{Resumo}

Introdução: Dentre as PICS empregadas em saúde, a terapia com florais de Bach mostra-se útil de diversas formas e para diversos fins, podendo ser aplicada, segundo alguns estudos, em várias especialidades incluindo a odontologia. Os Florais de Bach, desenvolvidos pelo médico inglês Dr. Edward Bach são substâncias extraídas de espécies de flores cuja vibração é compatível com algum tipo de disfunção que afeta a saúde dos indivíduos. No total foram extraídos 38 florais os quais foram distribuídos em sete grupos de acordo com sua finalidade. Objetivo: Avaliar o emprego dos florais de Bach na odontologia. Material e método: Trata-se de uma revisão narrativa de literatura. Os dados foram coletados durante o mês de outubro de 2019 nas plataformas Google Acadêmico, SciELO, e PubMed utilizando os descritores: Florais de Bach, odontologia, terapia. Os artigos que preencheram os critérios de elegibilidade foram selecionados e analisados. Resultados e discussão: Apesar de poucas pesquisas sobre o tema, sabe-se que os Florais de Bach são uma excelente opção no tratamento de várias condições psíquicas e físicas que aparecem no dia-a-dia da clínica odontológica. Estudos mostram que seu principal uso na odontologia se dá no combate do medo e ansiedade, no alívio de dores em lesões bucais e ainda é um potente antimicrobiano no combate do streptococoos mutans. Conclusão: Conclui-se que além de uma ótima opção terapêutica, os florais possuem propriedades as quais podem ser aplicadas em diversas áreas no tratamento odontológico, oferecendo uma eficácia excelente, custo acessível, facilidade de utilização, assim, otimizando a prática clinica.

Descritores: Odontologia; Terapêutica; Essências Florais.

\section{Abstract}

Introduction: Among the PICS employed in health, Bach flower therapy is useful in many ways and for various purposes and can be applied, according to some studies, in various specialties including dentistry. Bach Florals, developed by the English physician Dr. Edward Bach are substances extracted from flower species whose vibration is compatible with some type of dysfunction that affects the health of individuals. A total of 38 flowers were extracted and divided into seven groups according to their purpose. Objective: To evaluate the use of Bach florals in dentistry. Material and method: This is a narrative literature review. Data were collected during October 2019 on Google Scholar, SciELO, and PubMed platforms using the descriptors: Bach Florals, dentistry, therapy. Articles that met the eligibility criteria were selected and analyzed. Results and discussion: Despite little research on the subject, it is known that Bach Florals are an excellent option in the treatment of various psychic and physical conditions that appear in the day-to-day dental clinic. Studies show that its main use in dentistry is to combat fear and anxiety, to relieve pain in oral lesions and is still a potent antimicrobial to combat streptococoos mutans. Conclusion: It is concluded that besides being a great therapeutic option, florals have properties that can be applied in various areas in dental treatment, offering excellent efficacy, affordable cost, ease of use, thus optimizing clinical practice.

Descriptors: Dentistry; Therapeutics; Flower Essences.

\section{Resumen}

Introducción: entre los PICS empleados en salud, la terapia de flores de Bach es útil de muchas maneras y para diversos fines y puede aplicarse, según algunos estudios, en diversas especialidades, incluida la odontología. Bach Florals, desarrollado por el médico inglés Dr. Edward Bach, son sustancias extraídas de especies de flores cuya vibración es compatible con algún tipo de disfunción que afecta la salud de las personas. Se extrajeron un total de 38 flores y se dividieron en siete grupos según su propósito. Objetivo: evaluar el uso de flores de Bach en odontología. Material y método: Esta es una revisión de literatura narrativa. Los datos se recopilaron durante octubre de 2019 en las plataformas Google Scholar, SciELO y PubMed utilizando los descriptores: Bach Florals, odontología, terapia. Se seleccionaron y analizaron los artículos que cumplían los criterios de elegibilidad. Resultados y discusión: a pesar de la poca investigación sobre el tema, se sabe que los florales de Bach son una excelente opción en el tratamiento de diversas afecciones psíquicas y físicas que aparecen en la clínica dental diaria. Los estudios demuestran que su uso principal en odontología es combatir el miedo y la ansiedad, aliviar el dolor en las lesiones orales y sigue siendo un potente antimicrobiano para combatir los estreptococos mutantes. Conclusión: se concluye que además de ser una gran opción terapéutica, los florales tienen propiedades que se pueden aplicar en diversas áreas en el tratamiento dental, ofreciendo una excelente eficacia, costo asequible, facilidad de uso, optimizando así la práctica clínica.

Descriptores: Odontología; Terapéutica; Esencias Florales.

INTRODUÇÃO

Práticas integrativas e complementares também chamadas PICS são um conjunto de práticas voltadas ao tratamento e manutenção da saúde que conta com vários tipos de terapias alternativas, integrativas e complementares buscando uma nova forma de Medicina para a população e usando recursos terapêuticos baseados em conhecimentos tradicionais e práticas milenares cujo objetivo não é substituir a medicina convencional, mas complementá-la tornando-a mais integrativa ${ }^{1}$.

Atualmente, o Sistema Único de Saúde (SUS) oferece, de forma integral e gratuita, 29 procedimentos de Práticas Integrativas e Complementares (PICS) nos quais são empregados na Atenção Básica (principal porta 
de entrada para o SUS) e são indicadas por profissionais específicos conforme as necessidades de cada caso $^{2,3}$. Evidências científicas têm mostrado os benefícios do tratamento integrado entre medicina convencional e práticas integrativas e complementares ${ }^{4}$.

Especificamente na odontologia, o Conselho Federal de Odontologia (CFO) buscou incluir práticas a fim de que o cirurgião-dentista fosse apto e ampliasse as possibilidades do atendimento odontológico ${ }^{5,6}$. Assim, de acordo com a Resolução CFO 82/2008 foram reconhecidas Acupuntura, Homeopatia, Fitoterapia, Terapia floral, Hipnose, Laserterapia $^{5,7}$.

Dentre as terapias florais empregadas, a terapia com florais de Bach se mostra bastante útil na Odontologia de diversas formas e para diversos fins, podendo ser aplicada em várias especialidades odontológicas6. Os Florais de Bach, desenvolvidos pelo médico inglês Dr. Edward Bach ao longo de sua profissão, são substâncias extraídas de determinadas espécies de flores cuja vibração é compatível com algum tipo de disfunção que afeta a saúde das pessoas e, no total foram extraídos 38 florais os quais foram distribuídos em sete grupos de acordo com sua finalidade ${ }^{8}$.

Este documento se trata de uma revisão bibliográfica sobre os Florais de Bach e sua utilização na odontologia, ressaltando seu uso individualizado em cada especialidade odontológica.

\section{MATERIAL E MÉTODO}

Esta pesquisa trata-se de uma revisão da literatura do tipo narrativa, no qual se realizou uma seleção de artigos científicos obtidos a partir das bases de dados: Lilacs (Centro Latino- Americano e do Caribe de Informação em Ciências da Saúde), MEDLINE (Medical Literature Analysis and Retrieval Sistem Online), BVS (Biblioteca Virtual em Saúde), SCIELO (Scientific Eletronic Library Online) e PubMED, além de monografias que atenderam aos critérios do tema abordado, apresentando informações fundamentadas e publicadas no período compreendido entre 2009 e 2019, com exceção de artigos bases que se apresentaram relevantes para o estudo.

O trabalho foi realizado de 20 de agosto a 30 de outubro de 2019. O método de pesquisa utilizado nas bases de dados se deu pelo emprego dos seguintes descritores isolados ou em combinação, nas linguagens português, inglês e espanhol: "Florais de Bach", "Terapia floral", "Odontologia", "Florais na Odontologia",
"Bach Floral", "Odontology", "Floral Therapy" e "Dentistry" obtendo-se um total de 984 artigos dos quais 15 foram selecionados como amostra por atenderem os critérios que os tornam uteis para essa revisão da literatura.

RESULTADOS E DISCUSSÃo

As Práticas Integrativas e

Complementares (PIC's), além de apresentar opções de tratamento mais acessíveis, ampliam as abordagens de cuidado e as possibilidades terapêuticas para os usuários, garantindo desta maneira, uma maior integralidade e resolutividade da atenção à saúde?

Os Florais de Bach são reconhecidos e recomendados como terapia complementar pela Organização Mundial da Saúde (OMS), sendo cada remédio floral utilizado para tratar uma determinada pessoa, numa condição individual, visando o combate do desequilibro emocional, que, posteriormente se manifesta na forma de doenças $^{9}$. O uso dos remédios florais difundiuse pelo mundo, em especial por serem excelentes opções para o autocuidado, por não possuírem efeitos colaterais e nem toxicidade, oferecendo segurança para aqueles que o ultilizam ${ }^{9}$.

No estudo de Salles et al. $^{8}$, onde o objetivo era a diminuição do medo e ansiedade, aplicou-se um teste de medição dos sintomas em questão e, posteriormente a ministração do floral de Bach do tipo Rescue composto pelas essências florais Impatiens, Clematis, Star of Bethlehem, Cherry Plum e Rock Rose e a partir disso comprovou-se que todos $(100 \%)$ os voluntários que receberam a dose do floral Rescue tiveram sua ansiedade diminuída. Foi visto que "a maioria das pessoas (80 \%) que fez uso das essências florais, reportou que mesmo diante de eventos estressantes, conseguiu manter a calma, com clareza de ideias e concentração ${ }^{8}$.

Além de propriedades eficazes no combate dos desequilíbrios emocionais, o estudo de Gonçalves et al. ${ }^{10}$ apontou a eficácia do Floral de Bach quando testada suas propriedades antimicrobianas, revelando um resultado positivo na inibição do crescimento do streptococcus mutans quando utilizado os florais do tipo Rock Rose, Cherry Plum, Crab Apple, Impatiens, Rescue e Brandy, por meio da técnica de microdiluição em placas, apresentando resultados satisfatórios quando comparados a clorexidina, que representa o padrão ouro de atividade antimicrobiana ${ }^{10}$.

Os estudos de García Milanés et al. ${ }^{11} \mathrm{e}$ Moreno Peña ${ }^{12}$ avaliaram a utilização dos florais na odontopediatria. O estudo de Moreno Peña ${ }^{12}$ 
(2016) investigou e concluiu a eficácia dos Florais de Bach, mostrando que $90 \%$ das crianças apresentaram uma diminuição considerável nos níveis de ansiedade e medo durante 0 tratamento odontológico. Estes estudos tem grande importância para a odontopediatria, pois apresenta uma opção eficaz que auxilia no tratamento de crianças, que, por muitas vezes é demasiado complicado para o cirurgião-dentista e sua equipe ${ }^{11,12}$.

O estudo de Sisto et al. ${ }^{13}$ mostra, mais uma vez, os benefícios do uso dos Florais de Bach, dessa vez utilizados para tratar a gengivoestomatite herpética, condição comum no dia-a-dia da clinica odontológica que, com o uso dos florais apresentaram a diminuição da dor em $50 \%$ dos pacientes nas primeiras 72 horas, e na primeira semana, $100 \%$ do participantes da pesquisa relatam não mais sentir dor nas lesões ${ }^{13}$. Fernandes Neto et al. ${ }^{14}$ destacam que a habilitação de cirurgiõesdentistas em terapia com florais de Bach, poderia, além de difundir essa terapêutica, proporcionar aos pacientes 0 acesso a esse tratamento.

\section{REFERÊNCIAS}

1. Brasil. Ministério da Saúde. Gabinete do Ministro. Portaria $n^{\circ} 702$, de 21 de março de 2018. Altera a Portaria de Consolidação $\mathrm{n}^{\circ}$ 2/GM/MS, de 28 de setembro de 2017, para incluir novas práticas na Política Nacional de Práticas Integrativas e Complementares PNPIC. Brasília: Ministério da Saúde, 2018b.

2. Brasil. Ministério da Saúde. Gabinete do Ministro. Portaria no 971 de 03 de maio de 2006. Aprova a Política Nacional de Práticas Integrativas e Complementares (PNPIC) no Sistema Único de Saúde. Brasília: Ministério da Saúde, 2006.

3. Santos T, Silva CM, Possuelo LG, Morsch LM. Práticas integrativas e complementares na atenção básica: qual 0 conhecimento, aceitação e interesse dos usuários de um município do interior do RS?. Anais do Salão de Ensino e de Extensão;2018:7.

4. Telesi Júnior E. Práticas integrativas e complementares em saúde, uma nova eficácia para o SUS. Estud av. 2016;30(86):99-112.

5. Conselho Federal de Odontologia (CFO). Resolução no 82 de 25 de setembro de 2008. Reconhece e regulamenta o uso pelo cirurgião-dentista de práticas integrativas e complementares à saúde bucal. Rio de Janeiro, 2008.

6. Facioli F, Soares AL, Nicolau RA. Terapia floral na odontologia no controle de medo $e$ ansiedade-revisão de literatura. XIV Encontro Latino Americano de Iniciação Científica e X
Encontro Latino Americano de PósGraduação-Universidade do Vale do Paraíba, 2010.

7. Bueno N, FeesA, Franco LLMM, Reis LBM. Práticas integrativas e complementares: implantação nos serviços público e privado de saúde na Odontologia. Anais da Jornada Odontológica de Anápolis-JOA, 2019.

8. Salles LF, Da Silva MJP. Efeito das essências florais em indivíduos ansiosos. Acta paul enferm; 2012;25(2):238-42.

9. De Morais, Luan Caio Andrade; De Araújo Costa, Osvaldo Irineu Lopes; Araújo, Lia. O uso dos Florais de Bach nos últimos 10 anos: uma revisão integrativa. Anais Congrepics. 2019.

10. Gonçalves LTC, Miranda CC, Monteiro ABS, Dipp EC, Camargo GACG. Avaliação da eficácia de florais de bach na inibição do crescimento de streptococcus mutans: estudo in vitro. Rev Bras Odontol. 2018;75:(Supl.2):8

11. García Milanés $M$, Corona Carpio MH, Martínez Ramos MR, Medina Magluen C, Bruzón VC. Comportamiento clínico del miedo infantil al estomatólogo con tratamiento de flores de Bach. Rev Cubana Estomatol. 2007;44(3)

12. Moreno Peña BL. Miedo y ansiedad tratado con flores de Bach en la Clínica de Odontopediatría [dissertação]. Monterrey: Universidad Autónoma de Nuevo León;2016.

13. Peña Sisto $M$, de Silva Milagros $C$, Peña Sisto LA, González HE, Villalona Rodríguez J. Flores de Bach para el tratamiento de niños con gingivoestomatitis herpética aguda. MEDISAN. 2019;23(5):778-90.

14. Fernandes Neto JA, Simões TMS, LacerdaSantos JT, Lira AMM, Catão MHCV. Habilitação em terapia floral para cirurgiõesdentistas: uma análise por estados e regiões brasileiras. Arch Health Invest. 2019;8(10): 576-79

\section{CONFLITO DE INTERESSES}

Os autores declaram não haver conflitos de interesse

\section{AUTOR PARA CORRESPONDÊNCIA}

\author{
Abrahão Alves de Oliveira Filho \\ Unidade Acadêmica de Ciências Biológicas do Centro \\ de Saúde e Tecnologia Rural da Universidade Federal \\ de Campina Grande \\ 58428-830 Campina Grande - PB, Brasil \\ E-mail: abrahao.farm@gmail.com
}

